\title{
PREVALENCIA DE INCONTINENCIA FECAL EN PERSONAS QUE ACUDEN A POLICLÍNICOS DE UN HOSPITAL UNIVERSITARIO*
}

\author{
Drs. Antonella Sanguineti M. ${ }^{1}$, Katya Carrillo G. ${ }^{2}$, Günther Bocic A. ${ }^{1}$, \\ Carlos Domínguez C. ${ }^{1}$, Javier Fernández M. ${ }^{2}$, Mario Abedrapo M. ${ }^{1}$, \\ Rodrigo Azolas M. ${ }^{1}$, José Luis Llanos B. ${ }^{1}$, Mauricio Díaz B. ${ }^{1}$ \\ 1 Departamento de Cirugía. \\ 2 Departamento de Dermatología. \\ Hospital Clínico Universidad de Chile. \\ Santiago, Chile.
}

\begin{abstract}
Frequency of fecal incontinence in ambulatory patients

Background: Fecal incontinence (FI) affects quality of life and is a cause embarrassment, hampering the collection of data about the problem. It is more common in older people and it is important to know risk the factors and scope of the problem. Aim: Determine the prevalence and risk factors associated to FI in a population of patients consulting in a health center. Material and Methods: A self-applied questionnaire was answered by a randomly selected sample of 1136 ambulatory patients aged $50 \pm 15$ years (59\% females), excluding those who consulted in colorectal surgery and gynecology. Demographic variables, obstetric history, history of anorectal surgery, anorectal symptoms, involuntary urine loss, and the FI Wexner or Cleveland Clinic Fecal Incontinence scores were recorded. Results: Thirty one percent of respondents had some degree of FI. In multivariate analysis presence of anorectal pain, urinary incontinence and number of vaginal deliveries were significantly associated with FI. Conclusions: Prevalence of FI in this study is similar, though slightly higher, to data reported in national and international publications.
\end{abstract}

Key words: Fecal Incontinence, urine incontinence, Wexner score.

\section{Resumen}

Introducción: La incontinencia fecal (IF) es una entidad que afecta la calidad de vida y produce vergüenza, esto hace difícil la obtención de datos fidedignos en cuanto a su prevalencia. Dado el aumento de la

*Recibido el 30 de enero de 2015 y aceptado para publicación el 30 de marzo de 2015.

Los autores no refieren conflictos de interés.

Correspondencia: Dra. Antonella Sanguineti M.

asanguineti@gmail.com

Dr. Gunther Bocic A.

drbocic@yahoo.com 
población mayor en nuestro país y su relación con la aparición de IF, es importante conocer los factores de riesgo y magnitud del problema, para así realizar prevención además de un diagnóstico y tratamiento precoz. Objetivo: Determinar la prevalencia y los factores asociados a IF en personas que acuden a un centro de salud por otra causa. Material y Método: Se realizó una encuesta autoaplicada en el Hospital Clínico de la Universidad de Chile, entre mayo y junio de 2012. La muestra se seleccionó en forma aleatoria incluyendo pacientes mayores de 20 años que acudieron a diversos policlínicos, excluyéndose los policlínicos de coloproctología y ginecología. Se registraron variables demográficas, antecedentes obstétricos, antecedentes quirúrgicos anorrectales, sintomatología anorrectal, pérdida involuntaria de orina, y la escala de incontinencia fecal de Wexner o CCFIS. Resultados: De un total de 1.136 individuos, 59,2\% eran mujeres y la edad promedio fue de 50,53 $\pm 15,49$ años. Un 31,07\% presentaba algún grado de IF. En el análisis multivariado la presencia de dolor e incontinencia de orina, así como el número de partos presentaron una asociación significativa con la IF. Conclusión: Las cifras de prevalencia de IF en este estudio son algo mayores con respecto a datos nacionales e internacionales.

Palabras clave: Incontinencia fecal, incontinencia urinaria, score de Wexner.

\section{Introducción}

Aunque existen numerosos estudios basados en incontinencia fecal (IF), no existe una definición uniforme para ésta. En 1971, Duthie ${ }^{1}$ la definió como "un paciente que refiere un control anal inadecuado en mayor o menor grado". En 1993, Jorge y Wexner ${ }^{2}$ definieron la IF como "la pérdida involuntaria de heces o el ensuciamiento, al menos dos veces al mes". Actualmente se puede considerar IF como "la incapacidad de postergar la defecación en forma voluntaria, produciéndose una pérdida recurrente e involuntaria de las heces (líquidas y/o sólidas) y/o gases a través del conducto anal"'s.

A pesar de ser una enfermedad benigna, la IF resulta ser una entidad nosológica no solamente invalidante con alteraciones importantes en la calidad de vida ${ }^{4-8}$, sino también motivo de vergüenza, por lo que los pacientes habitualmente no la expresan de manera espontánea en la consulta médica9 ${ }^{9}$. Como consecuencia es probable que los datos publicados subestimen su prevalencia.

En la literatura internacional existe una gran variabilidad y discordancia con respecto al tema, dependiendo de la población y método de estudio utilizado, sin embargo, un rango entre 2 y $18 \%$ en población general es lo más aceptado ${ }^{4,10-17}$. Encontramos un estudio de Zárate et al., del año 2008, que presenta una prevalencia de IF a gases de un $20,5 \%$ y a sólidos y líquidos de un $2,7 \%{ }^{18}$. Esta experiencia se realizó en una población de pacientes mayores e institucionalizados, por lo que probablemente no representa lo que ocurre en la población general. No existen otros reportes nacionales que evalúen la prevalencia de IF. El objetivo de este trabajo es determinar la prevalencia y los factores asociados a IF en personas que acuden a un centro de salud por motivos diferentes a dicha patología.

\section{Material y Método}

Corresponde a un estudio observacional transversal realizado en nuestro hospital entre mayo y junio del año 2012. Se definió un tamaño muestral mínimo de 1.057 personas para tener estadísticamente un error de estimación de $3 \%$ y un intervalo de confianza de $95 \%$ (IC 95\%).

La selección de pacientes se realizó de manera aleatoria, donde se incluyó a los mayores de 20 años que acudían al policlínico del hospital. Fueron excluidos de esta muestra aquellos que consultaban por patología coloproctológica y/o ginecológica, pues se estimó que en este grupo podría existir un sesgo ya que existía una posibilidad bastante alta que consultaran por IF.

El estudio se realizó mediante una encuesta autoaplicada. Se registraron variables demográficas, cirugías anorrectales, sintomatología proctológica, presencia de incontinencia urinaria; para evaluar la IF se utilizó el Score de Wexner (SW) o CCFIS (Cleveland Clinic Fecal Incontinence Score) (Figura 1), de manera adicional en las mujeres fueron evaluados los antecedentes obstétricos.

Se definió como incontinencia urinaria a la pérdida involuntaria al menos una vez por semana e incontinencia fecal, como al escape de gases, deposiciones líquidas y/o sólidas de forma involuntaria por el ano con al menos un evento por semana.

Se explicó a los pacientes el tipo de encuesta y posteriormente se obtuvo el consentimiento informado. En cada caso en particular se aseguró el resguardo de la confidencialidad y el completo anonimato de los datos obtenidos, siendo utilizados únicamente para los objetivos ya descritos.

El grupo de estudio fue dividido en aquellos que no presentaban IF y los que sí la tenían y estos últimos se separaron según fuera a gases exclusivamente o a sólido-líquidos. Además se analizó los 


\begin{tabular}{|l|c|c|c|c|c|}
\hline Tipo IF & Nunca & $\begin{array}{c}\text { Raramente } \\
(<\mathbf{1} \text { vez/mes })\end{array}$ & $\begin{array}{c}\text { Algunas veces } \\
(>\mathbf{1} \text { vez/mes y } \\
<\mathbf{1} \text { vez/semana })\end{array}$ & $\begin{array}{c}\text { Frecuente } \\
(>\mathbf{1} \text { vez/semana } \\
\mathbf{y}<\mathbf{1} \text { vez/día })\end{array}$ & $\begin{array}{c}\text { Siempre } \\
(>\mathbf{1} \text { vez/día })\end{array}$ \\
\hline Gases & 0 & 1 & 2 & 3 & 4 \\
\hline Líquidas & 0 & 1 & 2 & 3 & 4 \\
\hline Sólidas & 0 & 1 & 2 & 3 & 4 \\
\hline Uso de paños & 0 & 1 & 2 & 3 & 4 \\
\hline $\begin{array}{l}\text { Alteración de } \\
\text { estilo de vida }\end{array}$ & 0 & 1 & 2 & 3 & \\
\hline & $\begin{array}{c}\text { 0: Continencia } \\
\text { perfecta }\end{array}$ & $20:$ & $\begin{array}{c}\text { Incontinencia } \\
\text { completa }\end{array}$ & & \\
\hline
\end{tabular}

Figura 1. Score de Wexner (español).

grupos con un SW mayor o igual a 9 puntos ya que éste representaría un punto de corte para un compromiso significativo de la calidad de vida como ha sido demostrado en estudios previos ${ }^{4}$.

En el análisis estadístico descriptivo, las variables cualitativas se representaron como porcentajes con un intervalo de confianza del 95\% (IC 95\%) y para las variables cuantitativas se evaluó la normalidad mediante la prueba de Shapiro-Wilk, y se representó mediante promedio y desviación estándar o mediana y rango intercuartil, según corresponda.

En el análisis estadístico se utilizó la prueba t de Student o test de Wilcoxon para variables cuantitativas según distribución, y test de Fisher o $\chi^{2}$ para las variables cualitativas.

Para evaluar la magnitud de la asociación de las variables se realizó regresión logística univariada y multivariada, determinándose el cálculo del Odds Ratios con un IC 95\%.

Se consideró un nivel de significancia del 0,05. El análisis estadístico se realizó con el programa SPSS ${ }^{\circledR}$ versión 20.0.0.

\section{Resultados}

Se presenta un total de 1.136 pacientes, de éstos 672 eran de sexo femenino $(59,2 \%)$, la edad promedio de la muestra fue de 50,53 $\pm 15,49$ años.

1. Del total de pacientes encuestados un $52,7 \%$ ha presentado IF a gases, líquidos o sólidos en alguna oportunidad y un $31,1 \%$ la presentó al menos una vez por semana, siendo este grupo el que se considera al realizar el análisis de este trabajo. En la mayoría de los casos fue sólo a gases $(89,9 \%)$ (Figura 2).

2. Los individuos que presentaron IF tenían mayor edad, síntomas anorrectales, antecedentes quirúrgicos proctológicos (específicamente hemo-

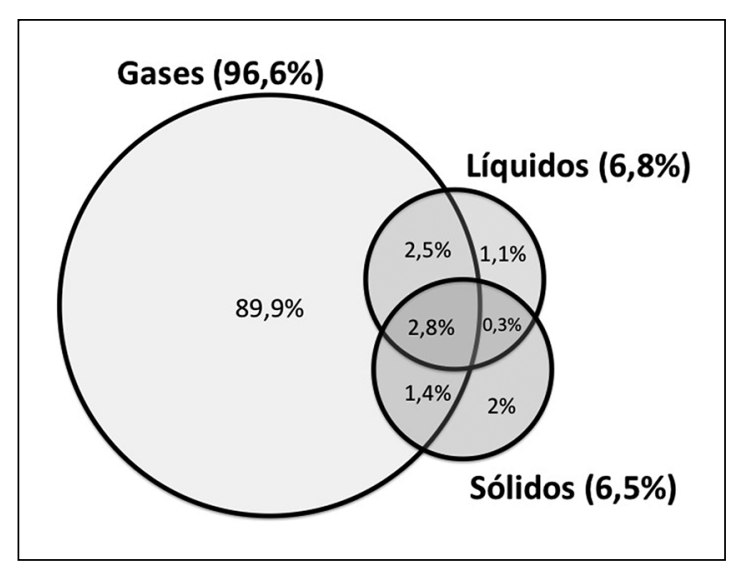

Figura 2. Gráfico de Venn. Distribución según tipo de IF.

rroides y fístulas). No hubo diferencias por sexo (Tabla 1).

3. El promedio de SW en los individuos con IF fue de $5 \pm 2,8$ puntos, sin diferencias por sexo. Los mayores de 50 años obtuvieron un SW mayor en comparación con los menores de 50 años $(5,25$ $\pm 3,1$ vs $4,63 \pm 2,32 ; \mathrm{p}=0,038$ ). Veintisiete pacientes obtuvieron SW mayor o igual a 9 puntos (7,64\%), de éstos $66,6 \%$ fueron mujeres. La presencia de incontinencia urinaria se asoció significativamente al grupo con SW mayor o igual a 9 puntos $(70,4 \%$ vs $24,5 \% ; \mathrm{p}<0,001$ (Tablas $2 \mathrm{y}$ $3)$.

4. En el análisis de regresión logística univariada, la presencia de IF mantuvo su asociación significativa con la edad (especialmente en los mayores de 40 años), la presencia de síntomas anorrectales (sangrado, prurito y/o dolor), los antecedentes quirúrgicos de hemorroides y fístula anal, y la presencia de incontinencia de orina. Sin embargo, 
Tabla 1. Variables estudiadas asociadas a IF $(n=1.136)$

\begin{tabular}{|lcccc|}
\hline & $\begin{array}{c}\text { Total } \\
(\mathbf{n}=\mathbf{1 . 1 3 6})\end{array}$ & $\begin{array}{c}\text { Sin IF } \\
(\mathbf{n}=\mathbf{7 8 3})\end{array}$ & $\begin{array}{c}\text { Con IF } \\
(\mathbf{n}=\mathbf{3 5 3})\end{array}$ & $\mathbf{p}$ \\
\hline Sexo & & & & \\
$\quad$ Femenino & $672(59,2 \%)$ & $451(57,6 \%)$ & $221(62,6 \%)$ & 0,118 \\
$\quad$ Masculino & $464(40,8 \%)$ & $332(42,4 \%)$ & $132(37,4 \%)$ & \\
Edad (años)* & $50,53 \pm 15,49$ & $48,98 \pm 15,19$ & $53,97 \pm 15,65$ & $<0,001$ \\
\hline Síntomas anorrectales* & $416(36,6 \%)$ & $258(33 \%)$ & $158(44,8 \%)$ & $<0,001$ \\
$\quad$ Sangrado* & $212(18,7 \%)$ & $131(16,7 \%)$ & $81(22,9 \%)$ & 0,014 \\
Prurito* & $103(9,1 \%)$ & $59(7,5 \%)$ & $44(12,5 \%)$ & 0,010 \\
Dolor* & $196(17,3 \%)$ & $112(14,3 \%)$ & $84(23,8 \%)$ & $<0,001$ \\
Cirugía proctológica* & $120(10,6 \%)$ & $71(9,1 \%)$ & $49(13,9 \%)$ & 0,017 \\
$\quad$ Hemorroides* & $72(6,3 \%)$ & $40(5,1 \%)$ & $32(9,1 \%)$ & 0,017 \\
Fisura anal & $25(2,2 \%)$ & $16(2 \%)$ & $9(2,5 \%)$ & 0,663 \\
Fístula anal* & $7(0,6 \%)$ & $2(0,3 \%)$ & $5(1,4 \%)$ & 0,033 \\
Prolapso rectal & $24(2,1 \%)$ & $16(2 \%)$ & $8(2,3 \%)$ & 0,825 \\
Incontinencia de orina* & $241(21,2 \%)$ & $142(18,1 \%)$ & $99(28 \%)$ & $<0,001$ \\
\hline
\end{tabular}

$* \mathrm{p}<0,05$.

Tabla 2. Análisis univariado de las variables estudiadas asociadas a IF

\begin{tabular}{|lllr|}
\hline & OR & CI 95\% & \multicolumn{1}{c|}{ p } \\
$\begin{array}{l}\text { Sexo } \\
\quad \text { Femenino }\end{array}$ & 1,23 & $0,95-1,6$ & 0,112 \\
$\quad$ Masculino & 1 & & \\
Edad (años)* & & & \\
$\quad$ Mayor de 40 años* & 1,67 & $1,23-2,27$ & 0,001 \\
Mayor de 50 años* & 1,61 & $1,25-2,08$ & $<0,001$ \\
Mayor de 60 años* & 1,58 & $1,21-2,06$ & 0,001 \\
Mayor de 70 años* & 1,88 & $1,31-2,7$ & 0,001 \\
Mayor de 80 años* & 3,13 & $1,55-6,3$ & 0,001 \\
Síntomas anorrectales* & 1,65 & $1,28-2,13$ & $<0,001$ \\
$\quad$ Sangrado* & 1,48 & $1,09-2,02$ & 0,013 \\
Prurito* & 1,75 & $1,16-2,64$ & 0,008 \\
Dolor* & 1,87 & $1,36-2,57$ & $<0,001$ \\
Cirugía proctológica** & 1,61 & $1,1-2,4$ & 0,015 \\
Hemorroides* & 1,85 & $1,14-3$ & 0,012 \\
Fisura anal & 1,25 & $0,55-2,87$ & 0,591 \\
Fístula anal* & 5,61 & $1,08-29,06$ & 0,040 \\
Prolapso rectal & 1,11 & $0,47-2,62$ & 0,809 \\
\hline Incontinencia de orina* & 1,76 & $1,31-2,36$ & $<0,001$ \\
\hline
\end{tabular}

$* \mathrm{p}<0,05$.

al someter estos valores al análisis multivariado, sólo la asociación con la presencia de dolor e incontinencia urinaria conservó significancia estadística (Tablas 2 y 3 ).

5. Un total de 317 pacientes presentaron IF exclusiva a gases $(27,9 \%)$, y 36 pacientes a sólido/ líquidos $(3,2 \%)$. Sólo la incontinencia de orina se
Tabla 3. Análisis multivariado de las variables estudiadas asociadas a IF

\begin{tabular}{|lllc|}
\hline & OR & \multicolumn{1}{c|}{ CI 95\% } & p \\
\hline Edad (años) & & & \\
Mayor de 40 años & 1,18 & $0,79-1,74$ & 0,407 \\
Mayor de 50 años & 1,16 & $0,79-1,72$ & 0,452 \\
Mayor de 60 años & 1,08 & $0,72-1,62$ & 0,712 \\
Mayor de 70 años & 1,51 & $0,96-2,37$ & 0,076 \\
Mayor de 80 años & 2,06 & $0,92-4,65$ & 0.079 \\
Síntomas anorrectales & 0,93 & $0,53-1,61$ & 0,782 \\
Sangrado & 1,35 & $0,82-2,24$ & 0,240 \\
Prurito & 1,54 & $0,91-2,6$ & 0,108 \\
Dolor* & 1,7 & $1,04-2,76$ & 0,034 \\
Cirugía proctológica & 0,64 & $0,31-1,3$ & 0,220 \\
Hemorroides & 2,11 & $0,92-4,83$ & 0,077 \\
Fístula anal & 5,8 & $0,96-35,2$ & 0,056 \\
Incontinencia de orina* & 1,49 & $1,09-2,03$ & 0,012 \\
\hline
\end{tabular}

$* \mathrm{p}<0,05$.

asoció en el análisis multivariado a la IF a sólido/ líquidos, siendo además la única variable que se asoció en el análisis multivariado a un SW mayor o igual a 9 (Tabla 4).

6. Las mujeres nulíparas fueron un $22,9 \%$, siendo este grupo significativamente de menor edad $(41,1 \pm 16,5$ vs $51,4 \pm 14,1$ años; $p<0,001)$. De las 518 mujeres que tuvieron al menos un parto, la mediana de partos vaginales fue 2 (RI: $0-3$ ), el $18,3 \%$ tuvo al menos un fórceps y un $24,9 \%$ tuvo un hijo de 4 kilos o más. 
Tabla 4. Variables estudiadas asociadas a IF según tipo: gases vs sólido/líquidos

\begin{tabular}{|c|c|c|c|}
\hline & $\begin{array}{l}\text { IF a gases } \\
(n=317)\end{array}$ & $\begin{array}{l}\text { IF a sólidos/líquidos } \\
\qquad(\mathrm{n}=\mathbf{3 6})\end{array}$ & $\mathbf{p}$ \\
\hline $\begin{array}{l}\text { Sexo } \\
\text { Femenino } \\
\text { Masculino }\end{array}$ & $\begin{array}{l}196(61,8 \%) \\
121(38,2 \%)\end{array}$ & $\begin{array}{l}25(60,4 \%) \\
11(30,6 \%)\end{array}$ & 0,371 \\
\hline Edad (años) & $54,1 \pm 15,7$ & $53,1 \pm 15,3$ & 0,712 \\
\hline $\begin{array}{l}\text { Síntomas anorrectales* } \\
\text { Sangrado } \\
\text { Prurito } \\
\text { Dolor* }\end{array}$ & $\begin{array}{r}135(42,6 \%) \\
69(21,8 \%) \\
39(12,3 \%) \\
70(22,1 \%)\end{array}$ & $\begin{array}{r}23(63,9 \%) \\
12(33,3 \%) \\
5(13,9 \%) \\
14(38,9 \%)\end{array}$ & $\begin{array}{l}0,015 \\
0,118 \\
0,785 \\
0,025\end{array}$ \\
\hline $\begin{array}{l}\text { Cirugía proctológica* } \\
\text { Hemorroides* } \\
\text { Fisura anal } \\
\text { Fístula anal } \\
\text { Prolapso rectal }\end{array}$ & $\begin{array}{c}40(12,6 \%) \\
29(72,5 \%) \\
7(17,5 \%) \\
4(10 \%) \\
5(12,5 \%)\end{array}$ & $\begin{array}{l}9(25 \%) \\
3(33,3 \%) \\
2(22,2 \%) \\
1(11,1 \%) \\
3(33,3 \%)\end{array}$ & $\begin{array}{l}0,042 \\
0,026 \\
0,741 \\
0,921 \\
0,127\end{array}$ \\
\hline Incontinencia de orina* & $81(25,6 \%)$ & $18(50 \%)$ & 0,002 \\
\hline
\end{tabular}

$* \mathrm{p}<0,05$.

Tabla 5. Análisis univariado de variables asociadas a IF, en mujeres según antecedentes obstétricos

\begin{tabular}{|lcccc|}
\hline & $\begin{array}{c}\text { Total } \\
(\mathbf{n}=\mathbf{6 7 2})\end{array}$ & $\begin{array}{c}\text { Sin IF } \\
(\mathbf{n = 4 5 1 )}\end{array}$ & $\begin{array}{c}\text { Con IF } \\
(\mathbf{n = 2 2 1})\end{array}$ & p \\
\hline Multíparas (cualquier vía) & $518(77,1 \%)$ & $342(50,9 \%)$ & $176(26,2 \%)$ & 0,270 \\
Partos Vaginales & $385(74,3 \%)$ & $254(74,3 \%)$ & $131(74,4 \%)$ & 0,968 \\
2 o más partos & $267(69,4 \%)$ & $172(67,7 \%)$ & $95(72,5 \%)$ & 0,333 \\
3 o más partos & $139(36,1 \%)$ & $85(33,5 \%)$ & $54(42,2 \%)$ & 0,133 \\
4 o más partos & $61(15,8 \%)$ & $32(12,6 \%)$ & $29(22,1 \%)$ & 0,015 \\
\hline Uso de fórceps & $95(18,3 \%)$ & $56(16,4 \%)$ & $39(22,2 \%)$ & 0,107 \\
RN macrosómico & $129(24,9 \%)$ & $77(22,5 \%)$ & $52(29,5 \%)$ & 0,080 \\
\hline
\end{tabular}

Porcentajes calculados sobre el total de multíparas.

Tener al menos un parto no se asoció a la presencia de IF, sin embargo, una cantidad mayor o igual a 4 partos vaginales fue la única variable que se asoció a mayor prevalencia de IF. Además el uso de fórceps se asoció a IF de tipo sólido/líquidos (32\% vs $17,6 \%, p=0,041)($ Tabla 5$)$.

\section{Discusión}

La IF resulta ser, sin duda, una entidad nosológica que está asociada a un desgaste sicológico muy significativo pues provoca una alteración en la calidad de vida del paciente y además resulta ser socialmente muy estigmatizada. Esto condiciona que poder obtener datos fiables sobre su prevalencia y/o factores asociados resulte ser bastante difícil y complicado ${ }^{9,10}$.
Los resultados de este trabajo coinciden con datos publicados a nivel mundial en que la edad, sobre todo en población mayor de 70 años, es un factor importante en la aparición de la $\mathrm{IF}^{19-21}$. Este dato debe ser especialmente considerado en la salud pública nacional puesto que para el año 2015 este grupo etario en nuestro país alcanzará el $4 \%$ del total de población según datos aportados por el Instituto Nacional de Estadísticas ${ }^{22}$.

En relación a las características específicas de esta patología la incontinencia a gases resulta ser mucho más frecuente que a sólidos y líquidos, lo que es similar con otras experiencias reportadas como la de Zárate et a $1^{18}$.

Existe coincidencia en la literatura médica nacional e internacional que en la población femenina la menopausia, con el consiguiente déficit en niveles hormonales, ocasiona con una frecuencia muy alta 
la presencia de una disfunción del piso pélvico. Por este motivo, no resulta extraño que la presencia de IF, así como sus formas más graves, (sólido/líquidos y $\mathrm{SW}>9$ puntos) se asocie en esta población de manera importante a la presencia de incontinencia urinaria ${ }^{23-25}$ reflejando así un posible compromiso global del piso pélvico.

También en pacientes femeninas existe el factor asociado a la maternidad y su consiguiente trabajo de parto, esto último ha sido ampliamente analizado y estudiado ${ }^{26,27}$. En nuestra experiencia la IF se relacionó solamente con el número de partos, pero no con el peso del neonato ni con el uso de fórceps lo que es coincidente con otras experiencias repor$\operatorname{tadas}^{28}$, este último se asocia a formas más graves de IF.

Pensamos, al igual que otros autores, que en este grupo poblacional la injuria obstétrica en realidad es multifactorial y se debe tener en cuenta el tipo de episiotomía, característica de la lesión del esfinter anal, daño del nervio pudendo, etc., lo que sumado al número de partos así como a la alteración del piso pélvico que se presenta posterior a la menopausia, sean la causa de la prevalencia significativa de IF en mujeres ${ }^{29}$.

De suyo resulta lógico esperar que la IF se relacionara con mayor frecuencia en pacientes que fueron sometidos a cirugía proctológica, lo que en nuestra experiencia resultó estar asociada a cirugía de fístula y hemorroides. Algunos tipos de fístulas pueden comprometer el aparato esfinteriano de manera muy compleja y su resolución quirúrgica debe estar en manos de un cirujano coloproctólogo, sin embargo, la realidad en nuestro país es que las operaciones de fístula no siempre las realiza un cirujano experto. En este punto, llama la atención que la cirugía de fisura no presente asociación con IF puesto que se reporta hasta un $15 \%$ de incontinencia a largo plazo posterior a la esfinterotomía lateral interna.

Respecto a la asociación de síntomas proctológicos y presencia de IF es difícil poder establecer si resultan ser causa o consecuencia. Es más lógico considerar la sintomatología referida por los pacientes como secundarias a la presencia de IF en mayor o menor grado y más significativos mientras más años de evolución tenga ésta.

La alta prevalencia y estigma social de la IF ha llevado a algunos autores a denominarla como "la epidemia silenciosa" y esto es similar en nuestro país. A la luz de los resultados encontrados en nuestra experiencia podemos establecer grupos de riesgo, así como recomendar una anamnesis muy dirigida a cada paciente preguntando directamente por la presencia de IF logrando un diagnóstico y tratamiento precoz, mejorando la calidad de vida de nuestros pacientes y evitando las morbilidades asociadas.

\section{Conclusión}

La prevalencia de incontinencia fecal es similar con tendencia a ser mayor a lo reportado previamente, sugiriendo una condición subdiagnosticada en nuestra población. Existe un porcentaje no menor de formas severas con posible repercusión en la calidad de vida, lo que transforma esta entidad en un problema de salud. La fisiopatología de la incontinencia fecal es compleja, diversos factores asociados han sido descritos, algunos no modificables como la edad, sin embargo, la presencia de factores modificables (número de partos, cirugías, etc.) deben ser sujetos de mayores estudios con el fin de disminuir la prevalencia de este problema.

\section{Referencias}

1. Duthie HL. Anal continence. Gut 1971;12:844-52.

2. Jorge JM, Wexner SD. Etiology and management of fecal incontinence. Dis Colon Rectum 1993;36:77-97.

3. Norton C, Whitehead WE, Bliss DZ, Tries J. Conservative and pharmacological management of faecal incontinence in adults. En: Incontinence. Abrams P, Cardozo L, Khoury S, Wein AJ. Ed. USA, Health Publications: Plymouth 2005; 1521-63.

4. Rothbarth J, Bemelman WA, Meijerink WJ, Stiggelbout AM, Zwinderman AH, Buyze-Westerweel ME, et al. What is the impact of fecal incontinence on quality of life? Dis Colon Rectum 2001;44:67-71.

5. Nelson R, Norton N, Cautley E, Furner S. Community-based prevalence of anal incontinence. JAMA 1995;274:559-61.

6. Deutekom M, Terra MP, Dobben AC, Dijkgraaf MG, Baeten CG, Stoker J, et al. Impact of faecal incontinence severity on health domains. Colorectal Dis. 2005;7:263-9.

7. Deutekom M, Terra MP, Dobben AC, Dijkgraaf MG, Baeten CG, Stoker J, et al. Impact of faecal incontinence severity on health domains. Impact of faecal incontinence severity on health domains. Colorectal Dis. 2005; 7:263-9.

8. Bordeianou LI, Rockwood T, Baxter N, Lowry A, Mellgren A, Parker S. Does incontinence severity correlate with quality of life? Prospective analysis of 502 consecutive patients. Colorectal Dis. 2008;10:273-9.

9. Alimohammadian M, Ahmadi B, Janani L, Mahjubi B. Suffering in silence: a community-based study of fecal incontinence in women. Int $\mathrm{J}$ Colorectal Dis. 2014;29:401-6.

10. Cotterill N, Norton C, Avery KN, Abrams P, Donovan JL. A patient-centered approach to developing a comprenhensive symptom and quality of life assessment of anal incontinence. Dis Colon Rectum 2008;51:82-7.

11. Lim JW, Heng C, Wong MT, Tang CL. Prevalence of 
faecal incontinence in the community: a cross-sectional study in Singapore. Singapore Med J. 2014;55:640-3.

12. Patel HD, Kamara TB, Kushner AL, Groen RS. Estimating the prevalence of urinary and fecal incontinence in a nationally representative survey in Sierra Leone. nt J Gynaecol Obstet. 2014;126:175-6.

13. Dunivan GC, Heymen S, Palsson OS, von Korff M, Turner MJ, Melville JL, et al. Fecal incontinence in primary care: prevalence, diagnosis, and health care utilization. Am J Obstet Gynecol. 2010;202(5):493.e1-6. doi:10.1016/j.ajog.2010.01.018. Epub 2010 Mar 12.

14. Jerez-Roig J, Souza DL, Amaral FL, Lima KC. Prevalence of fecal incontinence (FI) and associated factors in institutionalized older adults. Arch Gerontol Geriatr. 2015;60:425-30.

15. Roslani AC, Ramakrishnan R, Azmi S, Arapoc DJ, Goh A. Prevalence of faecal incontinence and its related factors among patients in a Malaysian academic setting. BMC Gastroenterol. 2014;14:95. doi: 10.1186/1471230X-14-95.

16. Giebel GD, Lefering R, Troidl H, Blöchl H. Prevalence of fecal incontinence: what can be expected? Int J Colorectal Dis. 1998;13:73-7.

17. Varma M, Brown J, Creasman J, Thom D, Van Den Eeden $\mathrm{S}$, Beattie, M, et al. Fecal Incontinence in Females Older Than Aged 40 Years: Who is at Risk? Dis Colon Rectum. 2006;49:841-51.

18. Zárate AJ, López-Köstner F, Vergara F, Badilla N, Viviani P. Prevalencia de la incontinencia fecal en centros de salud y casas de reposo. Rev Med Chile 2008;136:86772.

19. Madoff RD, Parker SC, Varma MG, Lowry AC. Faecal incontinence in adults. Lancet 2004;364:621-32.

20. Chassagne P, Landrin I, Neveu C, et al. Fecal incontinence in the institutionalized elderly: incidence, risk factors, and prognosis. Am J Med 1999;106:185-90.
21. Alessica P, Ouslander JG, Maldague S, Al-Samarrai NR, Saliba D, Osterweild W, et al. Incidence and costs of acute medical conditions in long-stay incontinent nursing home residents. J Am Med Dir Assoc. 2002;3:22942 .

22. www.ine.cl/canales/chile_estadistico/estadisticas_sociales_culturales/adultosmayores/pdf/mayorescenso2002. pdf.

23. Ditah I, Devaki P, Luma HN, Ditah C, Njei B, Jaiyeoba $\mathrm{C}$, et al, Prevalence, trends, and risk factors for fecal incontinence in United States adults, 2005-2010. Clinical Gastroenterology and Hepatology 2014;12:636-43.

24. Aslan E, Beji NK, Erkan HA, Yalcin O, Gungor F. The prevalence of and the related factors for urinary and fecal incontinence among older residing in nursing homes. Journal of Clinical Nursing 2009;18:3290-8.

25. Yip SO, Dick MA, McPencow AM, Martin DK, Ciarleglio MM, Erekson EA. The association between urinary and fecal incontinence and social isolation in older women. Am J Obstet Gynecol. 2013;208(2):146. e1-7. doi: 10.1016/j.ajog.2012.11.010. Epub 2012 Nov 15.

26. Oberwalder M, Connor J, Wexner SD. Meta-analysis to determine the incidence of obstetric anal sphincter damage. Br J Surg. 2003;90:1333-7.

27. Dudding TC, Vaizey CJ, Kamm MA. Obstetric anal sphincter injury: incidence, risk factors, and management. Ann Surg. 2008;247:224-37.

28. Johnson JK, Lindow SW, Duthie GS. The prevalence of occult obstetric anal sphincter injury following childbirth-literature review. J Matern Fetal Neonatal Med. 2007;20:547-54.

29. Parés D, Iglesias M, Pera M, Pascual M, Torner A, Baró $\mathrm{T}$, et al. Expression of estrogen and progesterone receptors in the anal canal of women according to age and menopause. Dis Colon Rectum 2010;53:1687-91. 